\title{
Dipolar vibrational modes in spherical semiconductor quantum dots
}

\author{
M. I. Vasilevskiy \\ Departamento de Física, Universidade do Minho, Campus de Gualtar, Braga 4710-057, Portugal
}

(Received 6 May 2002; published 27 November 2002)

\begin{abstract}
Spatially quantized dipolar phonon modes in a spherical quantum dot (QD) made of a polar isotropic material are considered in the framework of a continnum model. Different mechanical boundary conditions are analyzed, which are shown to strongly influence the spectrum of the dipole-active modes. The phonon-related polarizability of a single QD and an average dielectric function of a composite containing QDs are calculated. Numerical results are presented for CdSe and InP dots. A strongly dipole-active gap mode is predicted for InP QDs embedded in a matrix with a defined range of dielectric constant. The effect of increasing QD concentration in ensembles is discussed in terms of the dipole-dipole interaction between the dots, which can result in their bulk-like FIR absorption spectra with a peak at the transverse optical (TO) phonon frequency instead of the Fröhlich frequency. It is suggested that similar effects might occur in individual microcrystals, which can explain their absorbtion of FIR radiation at the TO phonon frequency, despite having a size much smaller than the radiation wavelength.
\end{abstract}

DOI: $10.1103 /$ PhysRevB.66.195326

PACS number(s): 78.30.-j, 63.22. $+\mathrm{m}$

\section{INTRODUCTION}

The effects of reduced space dimensions on the vibrational modes in quantum dots (QDs) are the subject of much current interest. ${ }^{1-10}$ Optical phonons confined in semiconductor QDs are important since they affect the electronic properties and are responsible for Raman scattering, which is one of the standard tools for the characterization of semiconductor structures. Most of the experimental studies of optical phonons in QDs have been performed by means of Raman spectroscopy. ${ }^{2,4-7,9,11,12}$ From the theoretical viewpoint, along with numerical lattice dynamics calculations for nanocrystals of some materials, ${ }^{13-15}$ a continuum model of Raman-active modes was developed for spherical QDs, ${ }^{16,17}$ which takes into account both mechanical and electrostatic confinement. It has been shown to give a good description of the experimentally observed spectra unless some further effects (such as disorder) are involved. ${ }^{2,9,18}$ However, to the best of my knowledge, nobody has succeeded to observe unambigously different quantized optical phonon modes resolved in Raman spectra. The reason is that, usually the highest frequency mode (with $l=0$ and $n=1$ in a spherical QD, where $n$ and $l$ are the spherical quantum numbers) is much more intense than the others, which, owing to the inevitable QD size dispersion, produce just an asymmetric broadening of the fundamental mode. Even if, under resonant conditions, two or three quantized modes have a comparable Raman scattering intensity, ${ }^{18}$ they are hard to resolve because of the small dispersion of the bulk optical phonon curves of the most of polar semiconductor materials.

Quantum-size effects on optical phonons can be better seen by far-infrared (FIR) absorption rather than Raman spectroscopy. As will be shown below, dipolar vibrational modes generally fill the whole range between the bulk transverse $\left(\omega_{T O}\right)$ and longitudinal $\left(\omega_{L O}\right)$ optical phonon frequencies. Although the absorption of, say, a micrometer-size sphere contains a single peak at the Fröhlich frequency, ${ }^{19}$ $\omega_{\mathrm{F}}=\left[\left(\epsilon_{s}^{\infty} \omega_{L O}^{2}+2 \epsilon_{h} \omega_{T O}^{2}\right) /\left(\epsilon_{s}^{\infty}+2 \epsilon_{h}\right)\right]^{1 / 2}$ (where $\epsilon_{s}^{\infty}$ and $\epsilon_{h}$ are the high-frequency dielectric constants of the sphere and surrounding medium), the situation changes when the mechanical confinement becomes important. As numerical lattice dynamics calculations show, ${ }^{20}$ several of the confined modes can produce significant FIR absorption in the limit of sufficiently small QDs. Even though there are relatively few FIR spectroscopy studies of phonons in QDs, ${ }^{1,8,10,20-22}$ the quantum-size effects indeed have been reported in some of them, including the observation of a well-resolved multimode structure in the transmittance spectra of thin films containing highly monodisperse CdSe QDs. ${ }^{8}$

Macroscopic approaches for optical phonons in semiconductor nanostructures are attractive because they give analytical results, which facilitates calculations of phononrelated observable properties. In the simplest approximation known as the dielectric continuum (DC) model, one solves the electrostatic equations for a (confined) polar medium with a frequency-dependent dielectric function describing its bulk phonon response. When applied to a spherical particle, this model predicts one surface (or interface) phonon mode for each number $l \geqslant 1$. (The $l=1$ one, with the frequency $\omega_{\mathrm{F}}$, is usually called the Fröhlich mode. ${ }^{19}$ ) For a semiconductor heterostructure, the DC model yields interface and confined phonon modes, both of which were observed experimentally. ${ }^{23}$ However, further experiments on shortperiod superlattices showed that the confined and interface modes intermix. Moreover, microscopic calculations ${ }^{24}$ demonstrated that the relative ionic displacement field is continuous across the interface, contrary to the results obtained from the DC model. A number of alternative macroscopic approaches were proposed, such as the hydrodynamics ${ }^{25}$ and modified DC ${ }^{26,27}$ models. The former generalized the classical Born and Huang theory ${ }^{28}$ of long-wavelength optical phonons in polar media by adding new terms in the equation of motion, which describe the effects of spatial dispersion. Unfortunately, owing to the mechanical boundary conditions (b.c.'s) chosen in Ref. 25, this model neglected the mixing of LO and TO phonon modes and produced results inconsistent with the experimental and numerical calculations. In the modified DC model, the confined phonons obey both elec- 
TABLE I. Material parameters used in the calculations.

\begin{tabular}{ccc}
\hline \hline \multicolumn{1}{c}{ Parameter } & CdSe & InP \\
\hline BZ center $\omega_{T O}, \mathrm{~cm}^{-1}$ & 169 & 307 \\
BZ center $\omega_{L O}, \mathrm{~cm}^{-1}$ & 211 & 347 \\
BZ edge $\omega_{T O}, \mathrm{~cm}^{-1}$ & 175 & 315 \\
BZ edge $\omega_{L O}, \mathrm{~cm}^{-1}$ & 152 & 330 \\
Damping, cm & 8 & 7.5 \\
$\epsilon_{s}^{\infty}$ & 6.2 & 5.7 \\
\hline \hline
\end{tabular}

trostatic and mechanical b.c.'s, that is, the relative ionic displacement is continuous across interfaces. The complete set of orthogonalized phonon modes in a heterostructure was first derived in Ref. 27, with the ionic displacement fields in good agreement with those obtained from the microscopic calculations. ${ }^{24}$ However, the confined phonons in this approach are dispersionless, i.e., all have the same frequency $\left(\omega_{L O}\right)$, therefore, the (modified) DC model is unsuitable for describing the fine structure of Raman or FIR spectra formed by several confined phonon modes with slightly different frequencies. The phonon dispersion has been incorporated in the model proposed in Refs. 29 and 30, which used the equations of motion of the hydrodynamic model ${ }^{25}$ but with different mechanical b.c.'s allowing the intermixing of confined $L O, T O$ and interface modes. This approach, consisting of solving the coupled continuous equation of motion and Poisson equation with b.c.'s specified in the next section, gives spectra of eigenmodes for semiconductor hetrostructures, which are very close to those of microscopic models, ${ }^{31}$ and has been succesfully applied for the calculation of Ramanactive modes in spherical QDs. ${ }^{16,17}$

In this work, a theory of dipole-active phonon modes in spherical QDs made of a polar material is presented, which is based on the approach proposed in Refs. 29 and 30. The theory presented below has allowed for a good description of the experimental results of Refs. 8 and 20. Apart from providing the details of the calculations (not published before), the following issues will be addressed: (i) a comparison of different mechanical boundary conditions; (ii) collective effects in QD ensembles and (iii) how the Fröhlich-type absorption is replaced by the bulk-like one when the crystallite size increases. The paper is organized as follows. In Sec. II, the principal equations are presented and quantized modes are obtained. In Sec. III, the observable quantities, such as effective dielectric function of a QD ensemble, are calculated. Section IV is devoted to a discussion and conclusions.

\section{QUANTIZED DIPOLAR MODES}

The phenomenological equation of motion has the following form: ${ }^{25,29,30}$

$$
\left(\omega^{2}-\omega_{T O}^{2}\right) \vec{u}=\beta_{L} \vec{\nabla}(\vec{\nabla} \vec{u})-\beta_{T} \vec{\nabla} \times(\vec{\nabla} \times \vec{u})+\frac{e_{T}}{\mu} \nabla \phi,
$$

where $\vec{u}$ is the relative displacement of the positive and negative ions, $\beta_{L}$ and $\beta_{T}$ are the curvature parameters of the bulk $L O$ and TO phonon dispersion curves, $e_{T}$
$=\sqrt{\epsilon_{S}^{\infty} \mu v\left(\omega_{T O}^{2}-\omega_{T O}^{2}\right) /(4 \pi)}$ is the transverse charge, $v$ the volume per cation-anion pair and $\mu$ is the reduced mass. The second equation, which relates the electrostatic potential, $\varphi$, to the mechanical displacement, reads as

$$
\vec{\nabla}\left(\epsilon_{s}^{\infty} \vec{\nabla} \varphi-\frac{4 \pi e_{T}}{v} \vec{u}\right)=0 .
$$

When applied to an infinite crystal, Eqs. (1) and (2) provide a reasonable (isotropic) approximation for its optical phonon dispersion curves. Contrary to Refs. 16 and 17, where $\beta_{L}$ and $\beta_{T}$ were taken constants measured in the Brillouin zone center, I considered them as frequency-dependent parameters defined in such a way that the $T O$ and $L O$ branches determined by Eqs. (1) and (2) coincide with the phonon dispersion curve of a diatomic linear chain,

$$
\omega^{2}(K)=\omega_{1}^{2}+\omega_{2}^{2}+\sqrt{\left(\omega_{1}^{2}+\omega_{2}^{2}\right)^{2}-4 \omega_{1}^{2} \omega_{2}^{2} \sin ^{2}(K / 2)},
$$

where $K$ varies between 0 and $\pi, \omega_{1}^{2}=\omega_{\min }^{2} / 2, \omega_{2}^{2}=\left(\omega_{\max }^{2}\right.$ $\left.-2 \omega_{1}^{2}\right) / 2$ and $\omega_{\min }$ and $\omega_{\max }$ are the minimum (Brillouin zone edge) and maximum ( $\Gamma$ point) $L O$ phonon frequencies (see Table I). This allows us to reproduce the zero group velocity and high density of states near the edges of the phonon bands, which is impossible with parabolic dispersion relations used in Refs. 16 and 17. For the TO branch with upward dispersion, I used the expression (3) with the opposite sign of the second term in the square root. Outside the allowed frequency band, $K$ was considered imaginary. The frequency-dependent bending parameters $\beta_{L}$ and $\beta_{T}$ were calculated as the corresponding derivatives, $-d \omega^{2} / d K^{2}$.

The spherically symmetric solution of Eqs. (1) and (2) is:

$$
\begin{gathered}
u^{r}=\left[A_{l} \frac{d}{d r} j_{l}(q r)+B_{l} l(l+1) \frac{g_{l}(k r)}{r}+C_{l} l r^{l-1}\right] Y_{l m}(\theta, \phi), \\
u^{\theta}=\left\{A_{l} j_{l}(q r)+B_{l} \frac{d}{d r}\left[g_{l}(k r) r\right]+C_{l} r^{l}\right\} \frac{1}{r} \frac{\partial}{\partial \theta} Y_{l m}(\theta, \phi),
\end{gathered}
$$

$$
u^{\varphi}=\{\cdots\} \frac{1}{r \sin \theta} \frac{\partial}{\partial \phi} Y_{l m}(\theta, \phi),
$$

where $q=\sqrt{\left(\omega_{L O}^{2}-\omega^{2}\right) / \beta_{L}}, k=\sqrt{\left|\left(\omega_{T O}^{2}-\omega^{2}\right) / \beta_{T}\right|}, j_{l}$ is the spherical Bessel function, $Y_{l m}$ the spherical harmonic and $g_{l}(x)$ is either $j_{l}(x)$ or $i^{-l} j_{l}(i x)$ depending on the sign of $\left[\left(\omega_{T O}^{2}-\omega^{2}\right) / \beta_{T}\right]$. [Note that the solution (4) does not include the torsional displacement component, ${ }^{32}$ which is decoupled from the other components and has no associated electric field). The constants $A, B$ and $C$, which determine the longitudinal, transverse and surface components of the confined vibration of a given frequency, are defined by b.c.'s. The complete set of b.c.'s includes ${ }^{29,30,32}$ (i) the continuity of the electrostatic potential and the normal component of the electric displacement, and (ii) the continuity of all components of $\vec{u}$ and components $\sigma^{i r}(i=r, \theta, \phi)$ of the stress tensor at the surface. The electrostatic b.c. (i) requires matching to the potential, $\bar{C}_{l} r^{-l-1}$, and electric field, $-\bar{C}_{l} \epsilon_{h}(l$ 


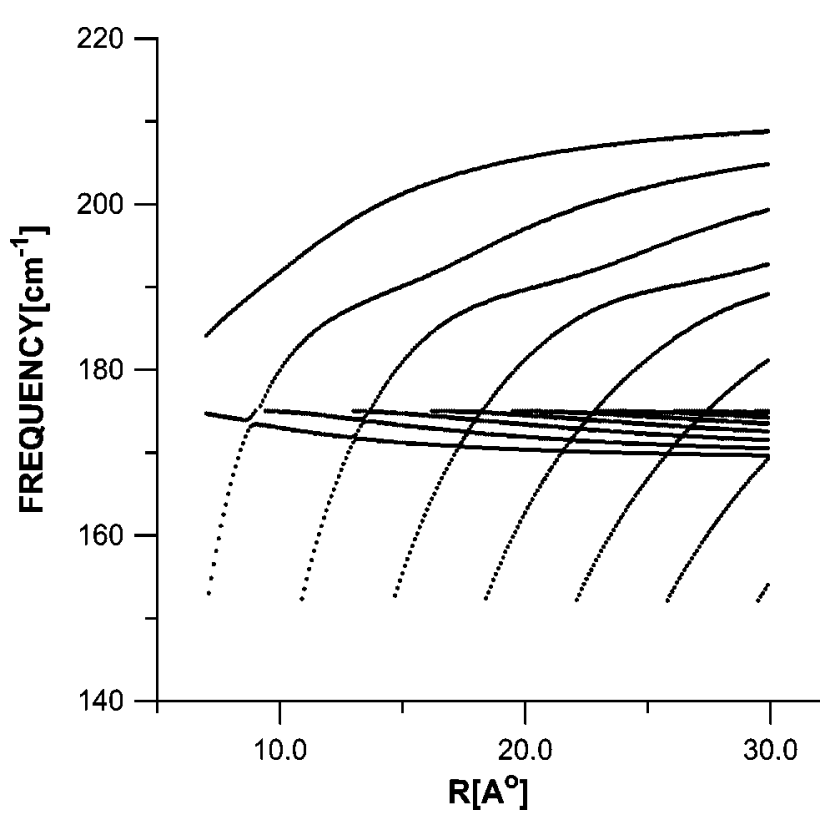

FIG. 1. The radial dependence of the $l=1$ phonon frequencies calculated for CdSe QDs embedded in a matrix with $\epsilon_{h}=3$ (rigid b.c.). The Fröhlich frequency is $192 \mathrm{~cm}^{-1}$.

$+1) r^{-l-2}$, outside the sphere. As to the mechanical b.c., I shall consider two limiting cases of (ii): (1) a rigid sphere ( $\vec{u}=0$ at the surface) and (2) a free-standing sphere $\left(\sigma^{i r}\right.$ $=0$ at the surface).

\section{A. Rigid b.c.}

The equation for the frequencies of the spheroidal modes of angular momentum $l$ is

$$
\begin{aligned}
& q R j_{l}^{\prime}(q R)\left\{\gamma \epsilon_{h}\left[k R g_{1}^{\prime}(k R)-l g_{1}(k R)\right]+\delta_{l}\left[k R g_{l}^{\prime}(k R)\right.\right. \\
& \left.\left.\quad+g_{l}(k R)\right]\right\}-l(l+1) j_{l}(q R)\left\{\gamma \epsilon_{h}\left[k R g_{l}^{\prime}(k R)-g_{l}(k R)\right]\right. \\
& \left.\quad+\delta_{l} g_{l}(k R)\right\}=0,
\end{aligned}
$$

where $\gamma=\left(\omega_{L O}^{2}-\omega_{T O}^{2}\right) /\left(\omega^{2}-\omega_{T O}^{2}\right), \quad \delta_{l}=\left[l \epsilon_{s}^{\infty}+(l+1) \epsilon_{h}\right]$ and $R$ is the QD radius. The dipolar $(l=1)$ mode frequencies calculated using Eq. (5) for CdSe QDs of different radii are plotted in Fig. 1. Note that none of them coincide with the Fröhlich frequency. The electrostatic Fröhlich mode corresponding to a uniform polarization of the sphere ${ }^{19}$ is not an eigenmode of the considered problem with rigid b.c. One can notice some bending in the dispersion curves of Fig. 1 near $\omega_{\mathrm{F}}$. The bending results in a small peak in the density of $l$ $=1$ states, defined as $\rho(\omega)=(1 / R) \Sigma_{n} \delta\left(\omega^{2}-\omega_{n}^{2}\right)$, shown calculated for a larger CdSe sphere in Fig. 2. The peak, apparently situated at the Fröhlich frequency, is formed by the modes whose frequencies are slighly lower or higher than $\omega_{\mathrm{F}}$.

The dependence of the mode frequencies on the QD radius calculated for most of the other $I I-V I$ and $I I I-V$ materials (whose bulk phonon dispersion curves are considered isotropic) is simlar to that shown in Fig. 1. However, InP is an interesting exception. The $L O$ and $T O$ phonon branches do not overlap in bulk $\mathrm{InP}^{33}$ so, there is a gap inside the optical

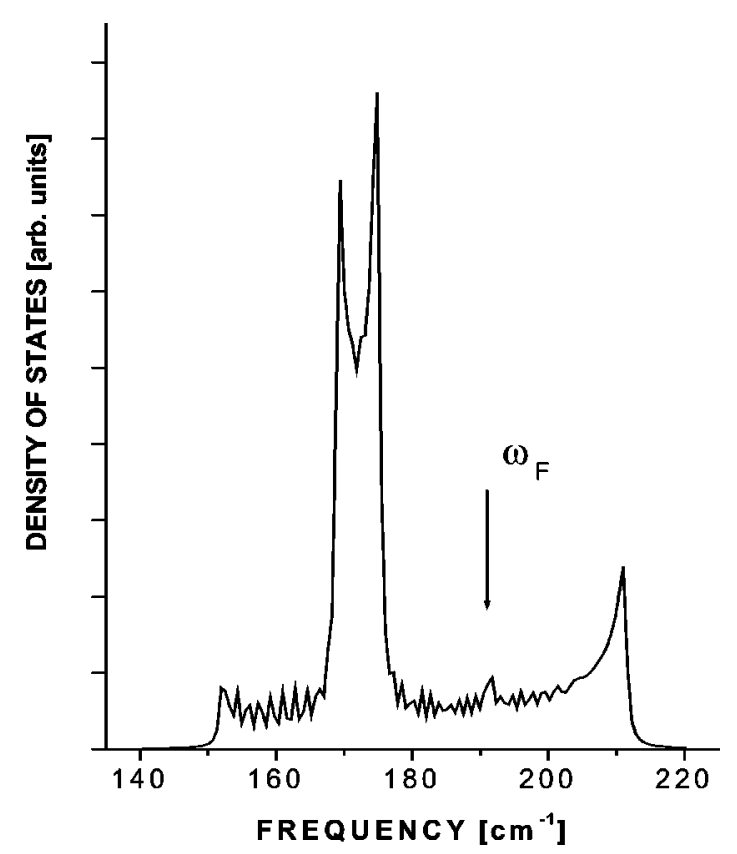

FIG. 2. Density of the $l=1$ phonon states calculated for a CdSe QD $\left[R=125 \mathrm{~nm}\right.$ embedded in a matrix with $\epsilon_{h}=3$ (rigid b.c.)].

phonon band. When $\epsilon_{h}$ is such that $\omega_{\mathrm{F}}$ falls inside the $L O$ or $T O$ band, the situation is not much different from that of CdSe (see Fig. 3, left). However, when $\omega_{F}$ occurs in the gap, a new feature appears, which is a gap mode of predominantly surface nature. The gap mode frequency is close to $\omega_{\mathrm{F}}$ and nearly independent of the QD size (see Fig. 3, right). As we will show, this mode, when it exists, should be dominating in the FIR absorption spectra.

\section{B. Free b.c.}

As it is shown in the Appendix, there are two kinds of $l$ $=1$ modes in this case: (i) the pure surface mode with $\omega$ $=\omega_{\mathrm{F}}$, and

(ii) mixed longitudinal-transverse-surface modes, whose frequencies obey the following equation:

$$
\begin{gathered}
4 \beta_{T}\left[q R j_{1}^{\prime}(q R)-j_{1}(q R)\right](k R)^{2} g_{1}(k R)+\beta_{L}(q R)^{2} j_{1}(q R) \\
\times\left[\left((k R)^{2}+2\right) g_{1}(k R)-2(k R) g_{1}^{\prime}(k R)\right]=0 .
\end{gathered}
$$

The modes determined by Eq. (6) do not produce any electric field outside the QD, therefore, they are not dipoleactive (although they can be Raman-active under certain conditions ${ }^{16}$ ). Therefore, the only dipole-active phonon mode in this case is the Fröhlich mode, which is characterized by the uniform displacement and polarization in the QD.

\section{FIR ABSORPTION}

\section{A. Polarizability of a single QD}

Let us consider a spherical QD subjected to an external electromagnetic field, $\vec{E}_{\text {ext }}$, of frequency $\omega$. The induced dipole moment of the sphere consists of two parts, $\vec{P}=\vec{P}_{0}$ $+\vec{P}_{1}$, where 


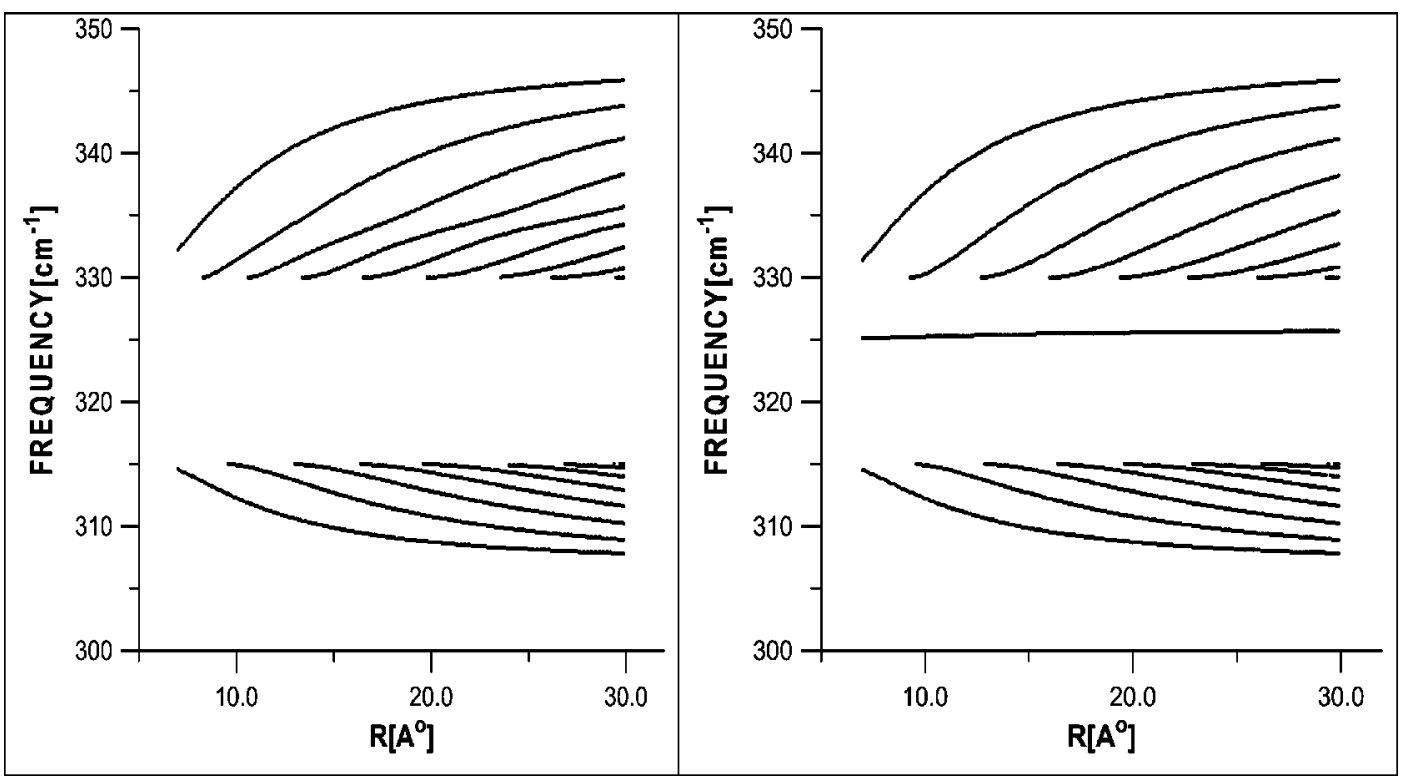

FIG. 3. The radial dependence of the $l=1$ phonon frequencies calculated for InP QDs embedded in a matrix with $\epsilon_{h}=1.5$ (left) and $\epsilon_{h}=4.5$ (right), assuming rigid b.c. The Fröhlich frequency is 336 and $326 \mathrm{~cm}^{-1}$, respectively.

$$
\vec{P}_{0}=\epsilon_{h} \frac{\epsilon_{s}^{\infty}-\epsilon_{h}}{\epsilon_{s}^{\infty}+2 \epsilon_{h}} R^{3} \vec{E}_{\mathrm{ext}}
$$

is a background polarization. The frequency-dependent part, $\vec{P}_{1}$, is due to the forced dipolar vibrations $\left(\vec{u}_{f}\right)$ induced in the sphere by the field,

$$
\vec{P}_{1}=-\frac{\partial}{\partial \vec{E}_{\mathrm{ext}}}\left(-\int\left(\vec{p} \cdot \vec{E}_{\mathrm{int}}\right) d \vec{r}\right)
$$

where $\vec{p}=\left(e_{T} / v\right) \vec{u}_{f}$ is the polarization vector and $\vec{E}_{\text {int }}$ $=3 \epsilon_{h} /\left(\epsilon_{s}^{\infty}+2 \epsilon_{h}\right) \vec{E}_{\text {ext }}$ is the field inside the sphere. The displacement $\vec{u}_{f}$ can be found through the Green's function of Eqs. (1) and (2),

$$
\begin{gathered}
\vec{u}_{f}=-\frac{v e_{T}}{\mu} \hat{G} \vec{E}_{\mathrm{int}}, \\
\hat{G}=\sum_{n, l, m} \frac{\vec{u}_{n l m}^{*} \otimes \vec{u}_{n l m}}{\omega^{2}-\omega_{n l m}^{2}+i \delta},
\end{gathered}
$$

where $\vec{u}_{n l m}$ are the eigenmodes discussed in the previous section, normalized by the condition $\int\left|\vec{u}_{n l m}\right|^{2} d \vec{r}=1$.

The QD polarizability is defined as $\left.\alpha=P /\left(\epsilon_{h} E_{\text {ext }}\right)\right) \equiv \alpha_{0}$ $+\alpha_{1}$. Combining Eqs. (8) and (9) and choosing the $z$ axis along $\vec{E}_{\text {ext }}$, we arrive to the following expression for its frequency-dependent part:

$$
\alpha_{1}=\frac{3 \epsilon_{s}^{\infty} \epsilon_{h}}{\left(\epsilon_{s}^{\infty}+2 \epsilon_{h}\right)^{2}}\left(\omega_{L O}^{2}-\omega_{T O}^{2}\right) \sum_{n} \frac{D_{n}^{2}}{\omega_{n}^{2}-\omega^{2}-i \delta},
$$

where

$$
\begin{aligned}
D_{n} & =(3 / 4 \pi)^{1 / 2} \int\left(\vec{u}_{n} \cdot \vec{e}_{z}\right) d \vec{r} \\
& =\int_{0}^{R}\left(u_{n}^{r}+2 u_{n}^{\theta}\right) r^{2} d r .
\end{aligned}
$$

In (10) and (11), the indices $l=1$ and $m=0$ have been omitted and $u_{n}^{r}$ and $u_{n}^{\theta}$ mean the radial dependence of the displacement components. The expression for the "background" polarizability $\alpha_{0}$ is obvious from Eq. (7).

The imaginary part of the polarizability obeys a certain sum rule. Since the coefficients $D$ are equal to zero for $l$ $\neq 1$ and (for the chosen field polarization) $m \neq 0$, we can write

$$
\sum_{n}\left|D_{n}\right|^{2}=(3 / 4 \pi) \int\left(\vec{e}_{z}\right)^{2} d \vec{r}=R^{3}
$$

Consequently,

$$
\int \operatorname{Im} \alpha d \omega^{2}=\pi R^{3} \frac{3 \epsilon_{s}^{\infty} \epsilon_{h}}{\left(\epsilon_{s}^{\infty}+2 \epsilon_{h}\right)^{2}}\left(\omega_{L O}^{2}-\omega_{T O}^{2}\right) .
$$

The integral (12) is equal to the area under the Lorentzian contour centred at the Fröhlich frequency, which corresponds to the only dipole-active mode allowed in a free-standing sphere. This latter case is perfectly described by the polarizabilty obtained by using the bulk dielectric function,

$$
\epsilon_{S}=\epsilon_{s}^{\infty}\left(1+\frac{\omega_{L O}^{2}-\omega_{T O}^{2}}{\omega_{T O}-\omega^{2}-i \delta}\right),
$$

for the QD, ${ }^{19}$

$$
\alpha=\frac{\epsilon_{s}-\epsilon_{h}}{\epsilon_{s}+2 \epsilon_{h}} R^{3} .
$$




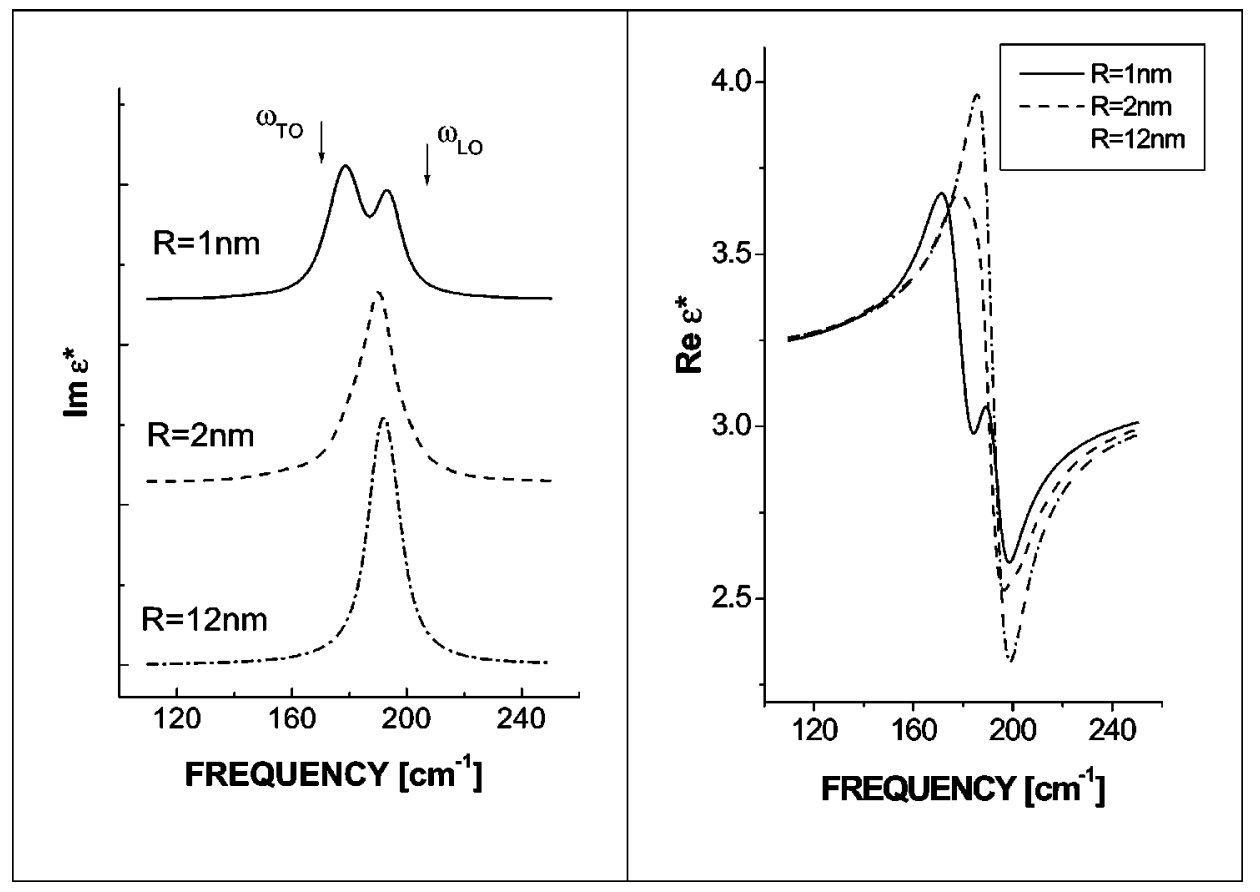

FIG. 4. Effective dielectric function (imaginary part-left; real part—right) of composites containing $f=0.05$ of CdSe QDs of different radii embedded in a matrix with $\epsilon_{h}=3$. A Gaussian distribution of sphere's radii was assumed, with a mean value as indicated in the figure and a relative standard deviation of $5 \% . \epsilon^{*}$ was calculated using the modified Maxwell-Garnett formalism and the single QD polarizability given by Eqs. (8), (10), (11).

Thus, there is no quantum size effect in the phonon-related dielectric response of a free-standing QD, which is perfectly described by the DC (electrostatic) model. ${ }^{19}$

On the contrary, several confined modes contribute to the polarizabilty of a QD embedded in a rigid matrix. This may result in a multimodal structure of the absorption of a system containing sufficiently small QDs. ${ }^{8,20}$ However, as we will see below, when the QD size increases, the net effect of the increasing number of allowed dipolar modes converges to that of the Fröhlich mode. Note that InP QDs, where the gap mode occurs, are an exception to this scenario. The calculations show that over $95 \%$ of the oscillator strength (11) is concentrated in the gap mode independently of the QD size.

\section{B. Dielectric function of a QD ensemble}

It is hardly possible to study a single QD by means of FIR spectroscopy. Since both the QD size and distance between them are much smaller than the FIR radiation wavelength, $\lambda$, the dielectric properties of a QD ensemble can be described by an effective (average) dielectric function, $\epsilon^{*}$. The FIR absorption of the ensemble can be calculated as $(2 \pi / \lambda) \operatorname{Im} \epsilon^{*} /\left(\operatorname{Re} \epsilon^{*}\right)^{1 / 2}$. Usually the QD filling fraction, $f$, of the experimentally studied materials does not exceed a few percent (except for so called close-packed QD films). Under these circumstances, the effective medium approach originally due to Maxwell-Garnett ${ }^{34}$ can be used with confidence to describe the average dielectric function. A version of this approach, which includes, to a certain extent, the dipole-dipole interaction between the polarized QDs and takes account of their size dispersion, ${ }^{35}$ was used in this work. The real and imaginary parts of $\epsilon^{*}$ calculated for composites containing an $f=0.05$ volume fraction of CdSe QDs of different sizes are shown in Fig. 4. One can see that the quantum confinement effect vanishes as the QD size increases.
If one wishes to describe the dielectric properties of a higher $f$ composite, the (modified) Maxwell-Garnett approximation is insufficient, because the dipole-dipole and higher multipole interactions between the dots become increasingly important. This problem was studied by many authors, including various approximate schemes (see Ref. 36 for a review) and an exact solution of the Maxwell equations for more than one sphere. ${ }^{37}$ It turns out that the classical meanfield equation,

$$
f \frac{\epsilon_{s}-\epsilon^{*}}{\epsilon^{*}+\frac{1}{3}\left(\epsilon_{s}-\epsilon^{*}\right)}+(1-f) \frac{\epsilon_{h}-\epsilon^{*}}{\epsilon^{*}+\frac{1}{3}\left(\epsilon_{h}-\epsilon^{*}\right)}=0,
$$

known as the Bruggeman approximation ${ }^{38}$ is the most popular, partly owing to its simplicity. Like the coherent potential approximation in the theory of electronic properties of alloys, ${ }^{39}$ it gives a good interpolation of the dielectric properties of a composite material between those of its end members although it may fail in reproducing details like spectral tails or fine structure. It is also known ${ }^{39}$ that, for a strong perturbation (of the dielectric function), such a mean-field approximation works well if the concentration of inclusions is not too small. Unfortunately, the inclusion of quantum-size effects in this formalism is not straightforward. As any approach designed to be valid for a wide filling fraction range including $f \approx 0.5$, the Bruggeman approximation treats both composite constituents on equal grounds. Therefore, one needs to define the dielectric function of the QD phase. A phenomenological way of doing this was suggested in Ref. 8 . If we equalize the polarizability given by the classical expression (14) to that given by formula (10) (with $\alpha_{0}$ added), we obtain 


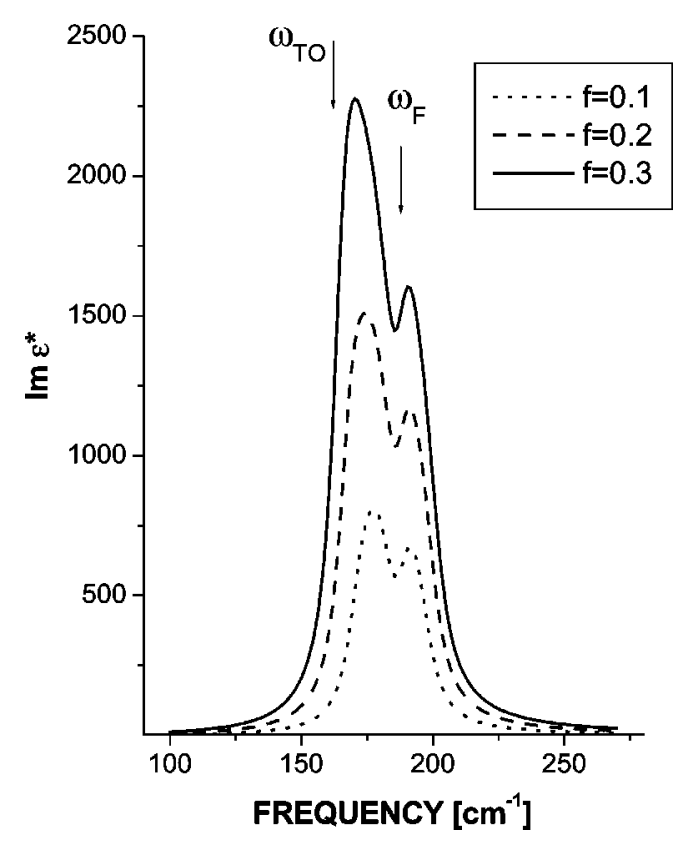

FIG. 5. Imaginary part of the effective dielectric function of composites containing $R=1 \mathrm{~nm}$ CdSe QDs embedded in a matrix with $\epsilon_{h}=3$, calculated using Eqs. (15), (16). The filling fraction is indicated in the figure.

$$
\epsilon_{s}=\epsilon_{s}^{\infty} \frac{1+\frac{8 \pi}{3}\left(\frac{\epsilon_{h}}{\epsilon_{s}^{\infty}}\right) \chi}{1-\frac{4 \pi}{3} \chi}
$$

where

$$
\chi=\frac{\left(\epsilon_{s}^{\infty}+2 \epsilon_{h}\right)}{3 \epsilon_{h}} \frac{\alpha_{1}}{\left(4 \pi R^{3} / 3\right)}
$$

If $\alpha_{1}$ contains a single resonance at the Fröhlich frequency, $\epsilon_{s}$ defined by Eq. (16) is just the bulk dielectric function
(13). The imaginary part of the effective dielectric function calculated using Eq. (15) for composites containing CdSe and InP QDs in high concentration is presented in Figs. 5, 6. Note that there is no quantum size effect in the case of InP QDs, which therefore were described by the bulk dielectric function (13).

\section{DISCUSSION AND CONCLUSIONS}

Results obtained in the previous section demonstrate that, for sufficiently small QDs embedded in a rigid matrix, the phonon-related FIR absorption can have a multimodal spectrum with several resonances corresponding to the few dipolar modes allowed in such a small sphere. When the QD size increases (above approximately $3 \mathrm{~nm}$ for $\mathrm{CdSe}$ and $\mathrm{CdS}$ ), the multimodal structure disappears and the absorption spectrum becomes "classical," with the only resonance at the Fröhlich mode. I would like to emphasize that, in fact, this is an apparent effect of many vibrational modes with the frequencies close to $\omega_{\mathrm{F}}$. Although the density of $l=1$ states has only a small peak at the Fröhlich frequency (Fig. 2), the modes closest to it have the largest oscillator strength. For example, in the case of InP QDs embedded in a matrix with an appropriate dielectric constant, the gap (surface) mode is by far the strongest in the FIR absorption. This effectively removes the quantum size effect for such dots. No quantum size effect should be expected also for free-standing QDs, where the size-independent Fröhlich mode is allowed.

There are few experimental observations of quantized dipolar phonon modes in QDs. ${ }^{1,8,20}$ One needs very small nanoparticles with a quite narrow size distribution. Nevertheless, using modern colloidal chemistry techniques, ${ }^{40-42}$ it is possible to obey these conditions. The experimentally observed multimodal structure in the absorption spectra of CdSe QDs ${ }^{8}$ was successfully described by the present theory. This means that the continuous model works well for dots as small as $2 \mathrm{~nm}$ in diameter and the lattice dynamics of such small crystallites has much in common with bulk crystals. The multimodal structure of the absorption also implies that

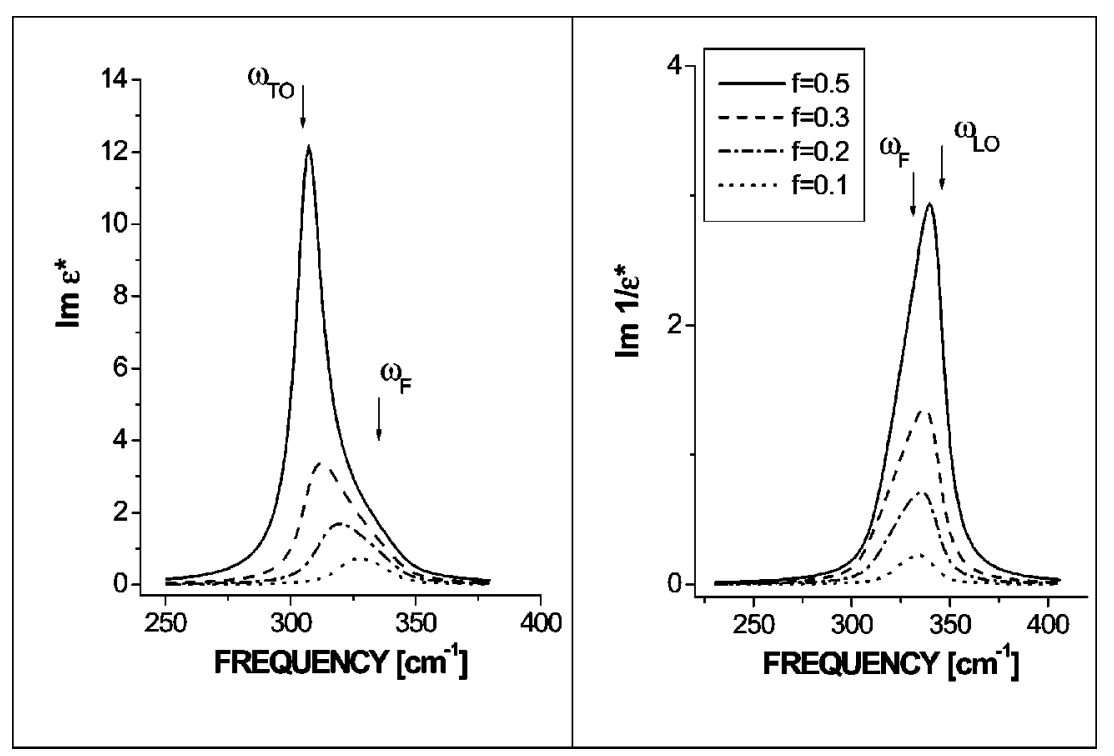

FIG. 6. Imaginary part of the effective dielectric function (left) and the dielectric loss function (right) of composites containing $R=1 \mathrm{~nm}$ InP QDs embedded in a matrix with $\epsilon_{h}=4.5$, calculated using Eqs. (15), (16). The filling fraction is indicated in the figure. 
rigid mechanical b.c. is a reasonable approximation to the reality. However, this may depend on the details of the nanocrystal fabrication technique. Chemically produced QDs always have a stabilizing organic shell, which prevents them from agglomeration in the colloidal solution. ${ }^{40-42}$ The CdSe QDs studied in Ref. 8 were capped with 1-thioglycerol and embedded in a polymethylmetacrilate (PMMA) matrix. ${ }^{41}$ Neither 1-thioglycerol no PMMA have detectable optical phonon modes in the reststrahlen band of $\mathrm{CdSe}^{43}$ which makes the rigid mechanical b.c. plausible in this case. Contrary to this result, just a single absorption peak between the bulk $T O$ and $L O$ phonon frequencies was observed in the spectra of CdS QDs capped with thiophenolate even for the QD size as small as $1 \mathrm{~nm},{ }^{21}$ which can be manifest because the free mechanical b.c. appears more relevant for this system. An interesting experiment was performed in Ref. 22, where the FIR spectra of embedded in a matrix and freestanding (i.e., obtained by chemically removing the matrix) CdS QDs were compared. While the spectrum of the freestanding QDs presented in Ref. 22 contains a single feature at the Fröhlich frequency, that of (exactly the same) QDs embedded in the matrix shows a weak structure. Probably the size uniformity of these QDs synthetized in a LangmuirBlogett film was insufficient to better resolve the phononrelated spectral structure.

Let us turn to the collective effects in the FIR absorption by QD ensembles. As any kind of interaction does, the dipole-dipole interaction between polarized QDs should split the resonant dipolar mode of a single QD. This effect was calculated explicitly for two touching spheres and for a linear chain of spheres, ${ }^{37}$ although in both cases the shift of the transverse dipolar mode with respect to the Fröhlich frequency did not exceed 5\%. If the single QD polarizability contains more than one resonance, increasing the QD filling fraction results not only in the mode's frequency shift but also in the increase of the relative intensity of the lower frequency mode (see Fig. 5). This concentration-dependent redistribution of the oscillator strength is also predicted by the modified Maxwell-Garnett theory. ${ }^{44}$ If the QD polarizability is given by the classical expression (14), the Bruggeman model predicts a gradual shift of the absorption peak from $\omega_{\mathrm{F}}$ to $\omega_{T O}$ when the QD concentration increases (Fig. 6 , left). At the same time, the loss peak shifts from $\omega_{\mathrm{F}}$ to $\omega_{L O}$ (Fig. 6, right). These peaks correspond to transverse and longitudinal collective excitations in the ensemble. Such a strong collective effect, which was not found for the linear chain of polarizable spheres ${ }^{37}$ is probably due to their threedimensional arrangement. The principal shortcoming of the Bruggeman model is the assumption of the topological equivalence of two ingredients of the composite medium. Nevertheless, I obtained similar concentration dependence of the absorption and loss peaks applying a different model, namely, a coherent potential approximation scheme developed for an ensemble of polarizable spheres randomly occupying the sites of a 3D lattice. ${ }^{45}$ It is beyond the scope of this paper to compare different models for calculation of the dielectric properties of composites. However, I agree with the authors of Ref. 46 that only experiments may decide which of the effective medium theories provides the best interpola-

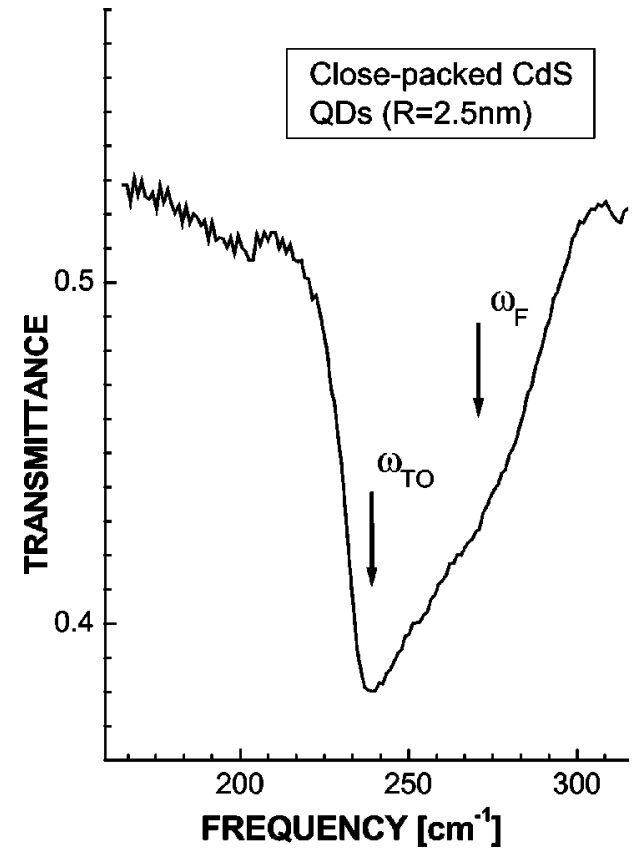

FIG. 7. Experimental transmittance spectrum of a thin matrixfree film of CdS QDs (Ref. 43). The Fröhlich $\left(\epsilon_{h}=1.5\right)$ and bulk $T O$ phonon frequencies are indicated.

tion between the properties of diluted composites and closepacked arrangements of polarizable spheres.

Experimentally, it is not easy to precisely control the QD filling fraction in composite films, partially because of the presence of the organic shell. The mean filling fraction of so called "close-packed QD films" studied in Refs. 8,20 and 41, in fact, did not exceed $30-35 \%$. Thus, it is hardly possible to study the concentration effect in QD ensembles quantitatively at this stage. However, it is noticeable that, in many cases, experimental FIR spectra of QD systems show a strong absorption peak approximately at the bulk $T O$ phonon frequency, instead of $\omega_{\mathrm{F}},{ }^{21}$ or a broad bulk-like reflection band extending from $\omega_{T O}$ to $\omega_{L O} \cdot{ }^{47}$ An example of such a spectrum is shown in Fig. 7. In my opinion, the only way to explain these and other similar results is to admit that the samples contained regions with elevated local QD concentration, which produced the absorption at $\omega_{T O}$ as predicted by the mean-field calculations (Fig. 6).

The last issue that I would like to discuss here concerns the transition from the Fröhlich mode to bulk polaritons when the crystal size increases. The electrostatic theory (see Refs. 19 and 48) predicts that this transition is governed by the value of $k_{0} R$, where $k_{0}=\epsilon_{h}^{1 / 2} \omega / c$ (typically $\sim 0.1 \mu \mathrm{m}^{-1}$ ). If $k_{0} R$ is small compared to unity, there are only small corrections to $\omega_{\mathrm{F}}$. Only when $k_{0} R \gg 1$, the bulk value of the TO-LO splitting is achieved. Nevertheless, experiments show an absorption peak at $\omega_{T O}$ and a longitudinal loss peak at $\omega_{L O}$ even for submicron polycrystalline $\mathrm{CdS}$ films. ${ }^{48}$ Moreover, a strong and sharp absorption peak at $\omega_{T O}$ (together with that at $\omega_{\mathrm{F}}$ ) was observed for $0.1 \mu \mathrm{m}$ crystallites of $\mathrm{MgO},{ }^{49}$ which were much larger than QDs considered in the present paper but still much smaller than the FIR wavelength. These particles should be perfectly described by 
the electrostatic theory because of their size and due to the fact that their concentration was just $3-4 \%$. So, the nature of the observed $T O$-like peak remained unexplained. I think this was also a dipole-dipole interaction effect similar to one discussed for QD ensembles. Owing to disorder, phonon polaritons are confined in small coherence regions within a microcrystal. Each coherence region (CR) is embedded in the matrix of the same material and closely surrounded by similar CRs. The electrostaic interaction between various CRs within a microcrystal produces the collective polariton mode at $\omega_{T O}$. This may happen even in relatively small microcrystals where $k_{0} R<1$, because there is no coherence between the displacements in different CRs implied in the consideration $^{19}$ of the polariton modes in a sphere.

In conclusion, quantized dipolar phonon modes in spherical QDs made of a polar semiconductor material were considered in the framework of a macroscopic model. These modes are presented calculated for CdSe and InP QDs subjected to rigid mechanical boundary conditions. A gap mode is predicted in the latter case for a certain range of values of the surrounding matrix dirlectric constant. Given the eigenmodes and eigenvectors, the phonon-related polarizability of a single QD can be calculated as explained in Sec. III A. There is a sum rule for the total oscillator strength of all quantized dipolar modes, which is equal to that of the Fröhlich mode, the only allowed dipole-active one for a freestanding QD. Moreover, the lineshape produced by all the allowed modes in a rigid-boundary QD is practically indistinguishable from the Fröhlich mode for $R>2 \mathrm{~nm}$ for typical $I I-V I$ and III-V materials. Increasing the concentration of QDs also leads to the smearing of the multimodal structure, if it exists for a single QD. However, the most important concentration effect is the increase of the apparent LO-TO splitting (i.e., the distance between the loss and absorption peaks). I suggest that this effect, owing to the dipole-dipole interaction between the polarized particles, is responsible for the unexpected experimentally observed absorption at $\omega_{T O}$ by small crystallites, including quantum dots.

\section{ACKNOWLEDGMENTS}

This work was supported by the Portuguese Foundation for Science and Techology (FCT) through Project No. POCTI/FIS/1012898.

\section{APPENDIX}

I consider here the $l=1$ spheroidal modes in a freestanding sphere. The necessary stress tensor components are

$$
\begin{gathered}
\sigma^{r r}=-2 \rho \beta_{T}\left\{A_{1}\left[\frac{d^{2} j_{l}(q r)}{d r^{2}}-\Lambda q^{2} j_{l}(q r)\right]\right. \\
\left.+2 B_{1} \frac{d}{d r}\left[\frac{g_{l}(k r)}{r}\right]\right\} Y_{l m}(\theta, \phi), \\
\sigma^{r \theta}=-\rho \beta_{T}\left\{2 A_{1} \frac{d}{d r}\left[j_{l}(q r) / r\right]\right. \\
\left.+B_{1} \frac{d^{2} g_{l}(k r)}{d r^{2}}\right\} \frac{\partial}{\partial \theta} Y_{l m}(\theta, \phi),
\end{gathered}
$$

where $\Lambda=\beta_{L} /\left(2 \beta_{T}\right)-1$. The $\sigma^{r \phi}$ component vanishes identically (it is nonzero only for the torsional modes not considered here). Putting $\sigma^{r r}=\sigma^{r \theta}=0$ at the sphere surface yelds Eq. (6).

The electrostatic b.c.'s result in the following equation:

$$
\begin{gathered}
\frac{4 \pi e_{T}}{v \epsilon_{s}^{\infty}}\left[2 \frac{B_{1}}{R} g_{1}(k R) \pm \frac{q^{2} \beta_{L}}{k^{2} \beta_{T}} \frac{A_{1}}{R} j_{1}(q R)\right] \\
=\frac{\bar{C}_{1}}{R^{3}} \frac{\left(\epsilon_{s}^{\infty}+2 \epsilon_{h}\right)}{\epsilon_{h}}\left(\frac{\omega_{\mathrm{F}}^{2}-\omega^{2}}{\omega_{T O}-\omega^{2}}\right),
\end{gathered}
$$

where \pm corresponds to the sign of $\left[\left(\omega_{T O}^{2}-\omega^{2}\right) / \beta_{T}\right]$ and $\bar{C}_{1}$ is the constant appearing in the expression for the potential outside the sphere (Sec. II). With the help of Eqs. (A1) for $r=R$, one can show that

$$
\left[-\beta_{L} q^{2} j_{1}(q R)\right] A_{1} \pm\left[2 \beta_{T} k^{2} g_{1}(k R)\right] B_{1}=0
$$

Then Eq. (A2) can be reduced to

$$
\bar{C}_{1}\left(\omega_{\mathrm{F}}^{2}-\omega^{2}\right)=0 .
$$

There are two possibilities to satisfy Eq. (A3), namely, (i) $\omega^{2}=\omega_{\mathrm{F}}^{2}, \bar{C}_{1} \neq 0, A_{1}=B_{1}=0$, and (ii) $\bar{C}_{1}=0, A_{1}, B_{1} \neq 0$. The former is, of course, the electrostatic Fröhlich mode. The frequencies of the modes (ii) are given by Eq. (6).
${ }^{1}$ T.D. Krauss, F.W. Wise, and D.B. Tanner, Phys. Rev. Lett. 76, 1376 (1996).

${ }^{2}$ C. Trallero-Giner, A. Debernardi, M. Cardona, E. MenendezProupin, and A.I. Ekimov, Phys. Rev. B 57, 4664 (1998).

${ }^{3}$ E. Menéndez-Proupin, C. Trallero-Giner, and S.E. Ulloa, Phys. Rev. B 60, 16747 (1999).

${ }^{4}$ A.V. Fedorov, A.V. Baranov, and K. Inoue, Phys. Rev. B 56, 7491 (1997)

${ }^{5}$ Y.N. Hwang, S.H. Park, and D. Kim, Phys. Rev. B 59, 7285 (1999).

${ }^{6}$ A. Ingale and K.C. Rustagi, Phys. Rev. B 58, 7197 (1998).
${ }^{7}$ A.G. Milekhin, A.I. Nikiforov, O.P. Pchelyakov, S. Schulze, and D.R.T. Zahn, JETP Lett. 73, 461 (2001).

${ }^{8}$ M.I. Vasilevskiy, A.G. Rolo, M.V. Artemyev, S.A. Filonovich, M.J.M. Gomes, and Yu.P. Rakovich, Phys. Status Solidi B 224, 599 (2001).

${ }^{9}$ M.I. Vasilevskiy, A.G. Rolo, M.J.M. Gomes, O.V. Vikhrova, and C. Ricolleau, J. Phys.: Condens. Matter 13, 3491 (2001).

${ }^{10}$ B. Zou, J. Wang, T. Green, and R. Little, Phys. Low-Dimens. Semicond. Struct. 5/6, 87 (2001).

${ }^{11}$ M.C. Klein, F. Hache, D. Ricard, and C. Flytzanis, Phys. Rev. B 42, 11123 (1990). 
${ }^{12}$ P. Tognini, L.C. Andreani, M. Geddo, A. Stella, P. Cheyssac, R. Kofman, and A. Migliori, Phys. Rev. B 53, 6992 (1996).

${ }^{13}$ J. Zi, M. Buscher, C. Falter, W. Ludwig, K. Zhang, and X. Xie, Appl. Phys. Lett. 69, 200 (1996).

${ }^{14}$ H. Fu, V. Ozolins, and A. Zunger, Phys. Rev. B 59, 2881 (1999).

${ }^{15}$ S.F. Ren, D. Lu, and G. Qin, Phys. Rev. B 63, 195315 (2001).

${ }^{16}$ M.P. Chamberlain, C. Trallero-Giner, and M. Cardona, Phys. Rev. B 51, 1680 (1995).

${ }^{17}$ E. Menendez, C. Trallero-Giner, and M. Cardona, Phys. Status Solidi B 199, 81 (1997).

${ }^{18}$ M.I. Vasilevskiy, A.G. Rolo, M.V. Artemyev, A. Mlyah, M.J.M. Gomes, and Yu.P. Rakovich, in Proceedings of the 25th International Conference on the Physics of Semiconductors, edited by N. Miura and T. Ando (Springer-Verlag, Berlin, 2001), p. 1209.

${ }^{19}$ R. Ruppin and R. Englman, Rep. Prog. Phys. 33, 149 (1970).

${ }^{20}$ A.G. Rolo, M.I. Vasilevskiy, N.P. Gaponik, A.L. Rogach, and M.J.M. Gomes, Phys. Status Solidi B 229, 433 (2002).

${ }^{21}$ T. Lover, G.A. Bowmaker, J.M. Seakins, and R.P. Cooney, Chem. Mater. 9, 967 (1997).

${ }^{22}$ A. Milekhin, M. Friedrich, D.R.T. Zahn, L. Sveshnikova, and S. Repinsky, Appl. Phys. A: Mater. Sci. Process. 69, 97 (1999).

${ }^{23}$ B. Jusserand and M. Cardona, in Nonlinear Optics of Random Media, edited by M. Cardona and G. Güntherodt (SpringerVerlag, Hedelberg, 1989).

${ }^{24}$ H. Rücker, E. Molinari, and P. Lugli, Phys. Rev. B 44, 3463 (1991).

${ }^{25}$ M. Babiker, J. Phys. C 19, 683 (1986).

${ }^{26}$ K. Huang and B. Zhu, Phys. Rev. B 38, 13377 (1988).

${ }^{27}$ R. Haupt and L. Wendler, Phys. Rev. B 44, 1850 (1991).

${ }^{28}$ M. Born and K. Huang, Dynamical Theory of Crystal Lattices (Clarendon, Oxford, 1986), Chap. 2.

${ }^{29}$ C. Trallero-Giner, F. Garcia-Moliner, V.R. Velasco, and M. Cardona, Phys. Rev. B 45, 11944 (1992).

${ }^{30}$ M.P. Chamberlain, M. Cardona, and B.K. Ridley, Phys. Rev. B
48, 14356 (1993).

${ }^{31}$ S.N. Klimin, E.P. Pokatilov, and V.M. Fomin, Phys. Status Solidi B 190, 441 (1995).

${ }^{32}$ E. Roca, C. Trallero-Giner, and M. Cardona, Phys. Rev. B 49, 13704 (1994).

${ }^{33}$ P.H. Borcherds, G.F. Alfrey, A.D.B. Woods, and D.H. Saunderson, J. Phys. C 8, 2022 (1975).

${ }^{34}$ J.C. Maxwell-Garnett, Philos. Trans. R. Soc. London 203, 385 (1904).

${ }^{35}$ M.I. Vasilevskiy and E.V. Anda, Phys. Rev. B 54, 5844 (1996).

${ }^{36}$ V.M. Shalaev, Nonlinear Optics of Random Media (SpringerVerlag, NY, 1999).

${ }^{37}$ J.M. Gérardy and M. Ausloos, Phys. Rev. B 22, 4950 (1980).

${ }^{38}$ D.A.G. Bruggeman, Ann. Phys. (Leipzig) 24, 636 (1935).

${ }^{39}$ J.M. Ziman, Models of Disorder (Cambridge University Press, Cambridge, 1979), Chap. 2.

${ }^{40}$ X. Peng, J. Wickham, and A.P. Alivisatos, J. Am. Chem. Soc. 120, 5343 (1998).

${ }^{41}$ M.V. Artemyev, A.I. Bibik, L.I. Gurinovich, S.V. Gaponenko, and U. Woggon, Phys. Rev. B 60, 1504 (1999).

${ }^{42}$ J. Rockenberger, L. Tröger, A.L. Rogach, M. Tischer, M. Grundmann, A. Eychmüller, and H. Weller, J. Chem. Phys. 108, 7807 (1998).

${ }^{43}$ A.G. Rolo, "Optical phonons in semiconductor quantum dots," Ph.D. thesis, University of Minho, Braga, Portugal, 2002.

${ }^{44}$ M.I. Vasilevskiy, Phys. Status Solidi B 219, 197 (2000).

${ }^{45}$ A. Leibsch and B.N.J. Persson, J. Phys. C 16, 5375 (1983).

${ }^{46}$ L. Genzel and U. Kreibig, Z. Phys. B: Condens. Matter 37, 93 (1980).

${ }^{47}$ M.I. Vasilevskiy, A.G. Rolo, and M.J.M. Gomes, Microelectron. Eng. 43-44, 715 (1998).

${ }^{48}$ M. Balkanski, in Photon-Phonon Interaction in Solids, edited by F. Abeles (North-Holland, Amsterdam, 1972), p. 541.

${ }^{49}$ L. Genzel and T.P. Martin, Phys. Status Solidi B 51, 91 (1972). 


\section{ERRATUM TO PAPER}

"Dipolar vibrational modes in spherical semiconductor quantum dots" by M I Vasilevskiy,

Phys. Rev. B 66, 195326 (2002)

Eq.(5) of the above paper contains some errors. The correct equation for the frequencies of the spheroidal modes of angular momentum $l$ is:

$$
\begin{gathered}
q R j_{l}^{\prime}(q R)\left\{\gamma \epsilon_{s}^{\infty}\left[-k R g_{l}^{\prime}(k R)+l g_{l}(k R)\right]+\delta_{l}\left[k R g_{l}^{\prime}(k R)+g_{l}(k R)\right]\right\} \\
-l(l+1) j_{l}(q R)\left\{\gamma \epsilon_{h}\left[k R g_{l}^{\prime}(k R)-l g_{l}(k R)\right]+\delta_{l} g_{l}(k R)\right\}=0
\end{gathered}
$$

The calculated results presented in the paper are correct. 